F2012-E12-015

\title{
DEVELOPMENT OF A VEHICLE SIMULATOR BASED ON A REAL CAR FOR RESEARCH AND EDUCATION PURPOSES
}

\author{
${ }^{*}$ Szalay, Zsolt; ${ }^{2}$ Gáspár, Péter; ${ }^{1}$ Kánya, Zoltán; ${ }^{2}$ Nagy, Dávid \\ ${ }^{1}$ Department of Automobiles, Budapest University of Technology and Economics, Hungary \\ ${ }^{2}$ Systems and Control Laboratory, Computer and Automation Research Institute, Hungary
}

KEYWORDS - vehicle simulator, embedded functions, driver assistance systems

ABSTRACT - The paper presents a hardware-in-the-loop simulation environment, which is built in such a way that the simulator tends to the real vehicle functions as much as possible. The simulation system contains several components such as an HMI (Human Machine Interface), a high-accuracy validated simulation software operated on a PC and a visual system with real-time graphics. The simulator is equally suitable for educational and research purposes. All the vehicle engineer students use the simulator system during their curriculum, enabling the thorough understanding of modern vehicle functions, thus improving the competence of future generations of engineers. Moreover the simulator system projects ahead the opportunity of new vehicle research that induces considerable additional scientific results.

\section{INTRODUCTION}

A vehicle simulator system which is based on a real car (Audi TT Coupe) is developed. The simulator system is unique because it uses a real car for receiving the input signals of the driver and displaying information about the simulation. The real car can be switched to simulation mode by a push-button switch. The system is safe: the additional electronic units are isolated galvanically from the original electrical system of the vehicle and in the simulation mode the vehicle engine cannot be started.

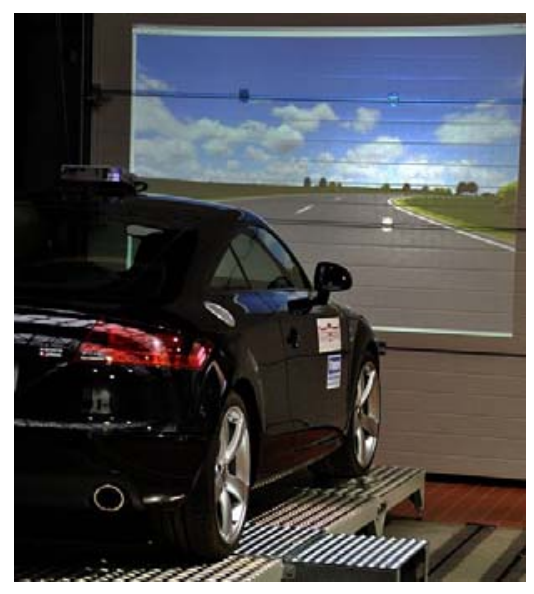

Figure 1: Vehicle simulator system

The purpose of the simulator system is twofold. One is the integration of the simulator system into the education of vehicle engineering. The simulator system is based on a real vehicle, and it uses high technology solutions which are applied in modern vehicles. 
Modern technology and the realistic driving experience of the simulator increase the motivation of the students, and the simulator system ensures the better understanding of the vehicle dynamics, vehicle control systems, the system design methods and the electrical, computational and communication systems. The modular and flexible structure of the system enables the implementation of a number of vehicle control systems on the simulator, and additional electronic control units (ECU) and sensors can also be installed. On the other hand, the vehicle simulator is also a basis for research activities. The achievements of vehicle control research can be demonstrated and tested on the system, and a number of measurements can be carried out on the simulator - e.g. for investigating driver behavior. Furthermore the modular structure ensures that optional equipment (e.g. additional ECUs, sensors or actuators) can be fitted into the system for running hardware-in-the-loop simulations.

\section{STRUCTURE OF THE VEHICLE SIMULATOR}

The main concept of the vehicle simulator is shown in Figure 2. The driver sits in the driver seat and controls the accelerator and brake pedals, the steering wheels and the transmission selector lever such as in real life, but the vehicle does not move. The simulation of the vehicle model and the environment (road, terrain, etc.) are run on the simulator PC. The simulation software calculates the vehicle motions by validated vehicle models then the scene is visualized in real time. The visualization of the vehicle and the environment are projected in front of the driver by a projector.

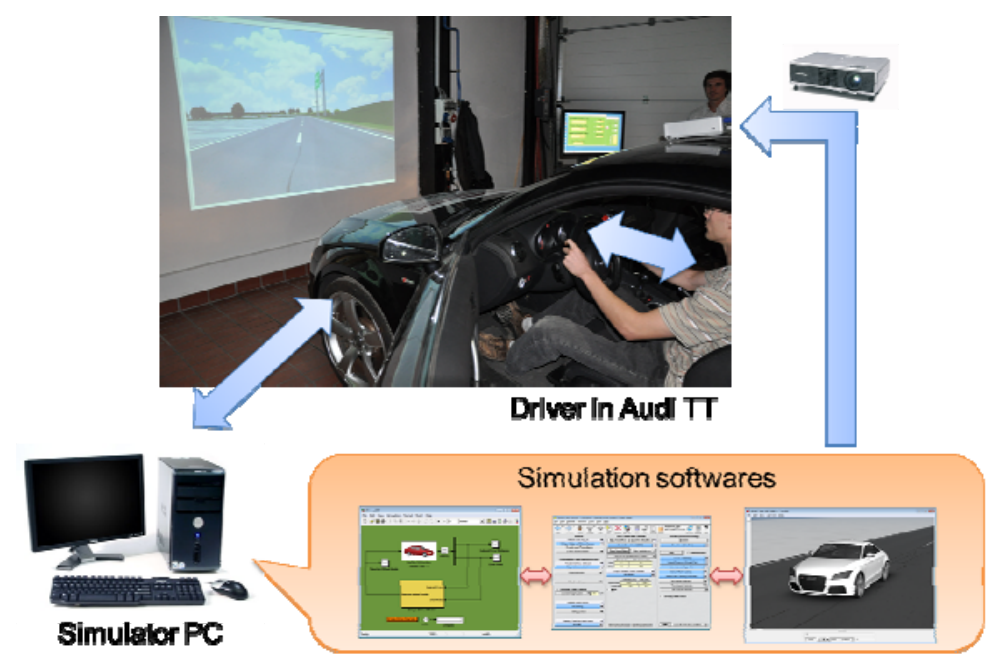

Figure 2: The concept of the vehicle simulator

The specialty of the system is that the driver's cabin of the simulator is set up on the base of a real car and with one single switch the simulator can be converted back to a conventional road vehicle. The challenge is to keep the original vehicle functions intact while implementing simulation functions. For this reason the control of the vehicle's communication network has been taken over by the vehicle control panel, implementing full RBS simulation for the instrument cluster in simulation mode. The standing vehicle can be 'driven' exactly the same way as on a test track: there is engine sound and screech while skidding; the dash panel displays the current speed and the engine rpm and one can shift gears just like in real life. 
The main advantage of keeping the original vehicle functions is that the system allows further educational and research opportunities. Keeping the moving capability of the vehicle enables real dynamic measurements on a test track, which induce more research projects; e.g. controloriented vehicle model identification tasks or implementation of novel vehicle dynamic control algorithms. Modular and multilevel system architectures are applied at the development of the simulator components for ensuring simple further extensions of the system.

The structure of the vehicle simulator system is shown in Figure 3. The simulation consists of two parts. One is the vehicle incorporating HMI (Human Machine Interface) functions, the other is a simulator application based on a PC which implements the physical model of the vehicle and the environment. As vehicle simulations are carried out with the engine switched off the missing internal signals are also generated in the vehicle to ensure the basic functions. During the modification of the electronic system of the vehicle crucial requirement was to provide plausible sensor signals which are necessary for control using the electronic systems of the real AUDI TT Coupe test vehicle. A standard (J1939), duplex and real-time CAN communication line was built between the vehicle control panel and the simulation PC. The simulation PC uses a standard CAN interface card for receiving and transmitting the specific input and output signals of the simulation.

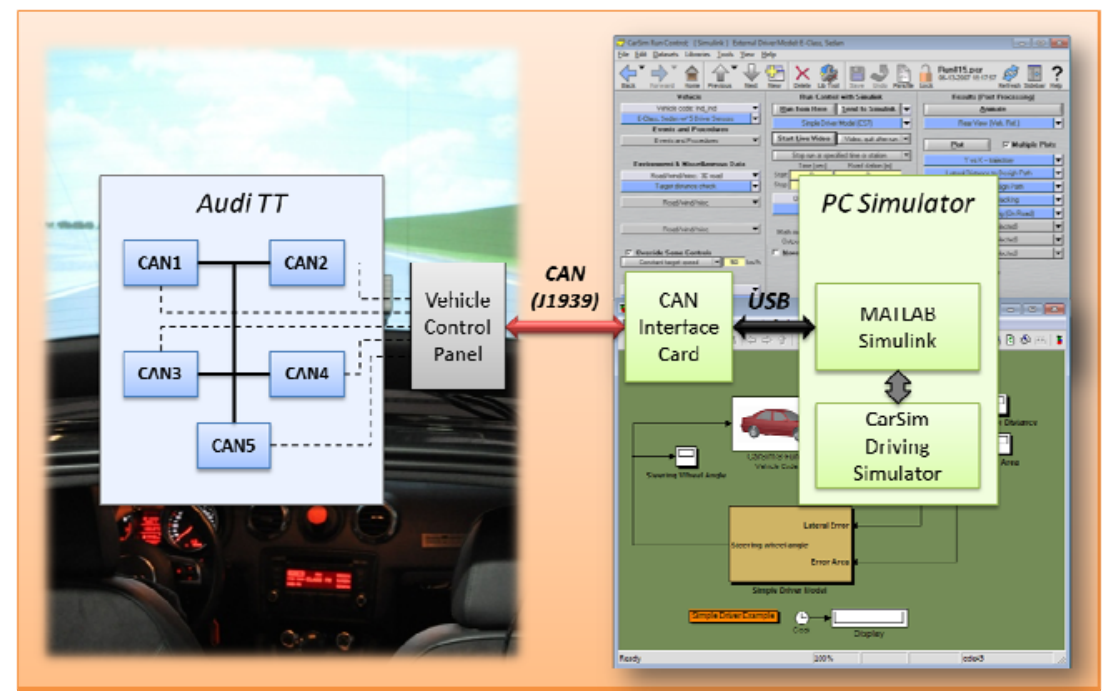

Figure 3: Structure of vehicle simulator

The driver can induce various vehicle maneuvers by using the steering wheel, the accelerator/brake pedals of the real car. The vehicle model and the simulation environment are built in the complex vehicle dynamics software CarSim. The models are implemented in MATLAB/Simulink environment because it enables the simple extension of the model with the CAN communication, the control systems and optional additional system models. The simulation is run in MATLAB/Simulink environment using the differential equation solvers of MATLAB. The MATLAB/Simulink simulation communicates with CarSim which visualizes the scene in quasi real-time. Based on the excitations and the control signals of the driver, the MATLAB/Simulink model generates the signals of the vehicle motions during the simulation. Then the CarSim Driving Simulator shows the vehicle maneuvers by quasi realtime graphics projected in front of the vehicle, and it provides the signals during the journey. Since the conditions of the simulation can change various simulation experiences are achieved. 


\section{VEHICLE CONTROL PANEL}

During the development of the vehicle control panel, it was a basic requirement to reach the essential sensor information of the electronic system of the vehicle. The specific signals (e.g. pedal and steering wheel positions) are sent to the simulation PC via the vehicle control panel. The control panel contains standard J1939 duplex CAN gateway electronics, and it is extended with other components which ensure the comfort and safe operation of the simulator system such as an engine sound generator and operation mode selector electronics. The cockpit and the vehicle control panel are shown in Figure 4.

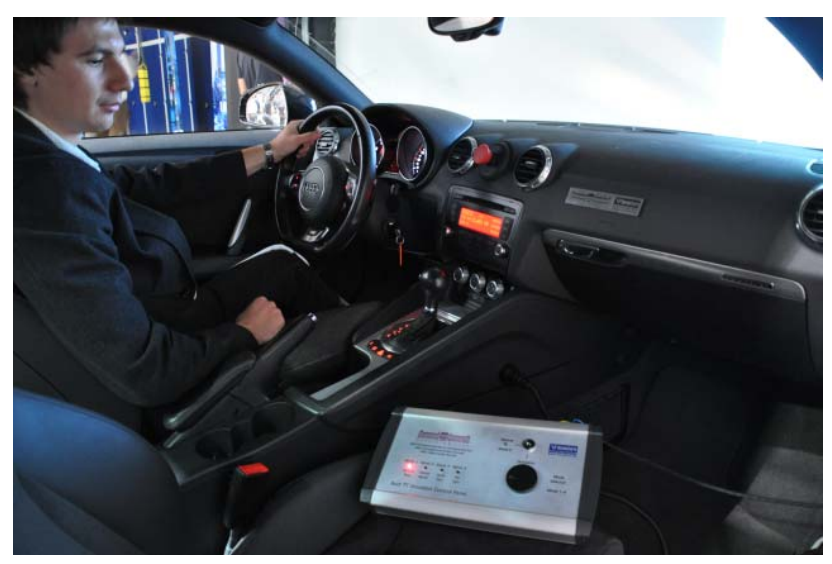

Figure 4: The cockpit and the vehicle control panel

Besides the gateway function, the other important task of the control panel is taking over the control of the vehicle dashboard in the simulation modes. It requires that the control panel simulate the full electronic system of the vehicle in order to display the vehicle speed, the engine rpm, the engine temperature and the actual gear on the dashboard without any error messages. The residual bus simulation (RBS) is implemented on the vehicle control panel, and it emulates a real vehicle electronic environment to the dashboard electronics. The vehicle control panel receives signals from the simulation PC via the CAN bus then displays them on the dashboard.

Furthermore the vehicle control panel ensures the safety of the system. In normal vehicle operating mode, it galvanically decouples the additionally installed electronic devices from the original electronic system of the vehicle. The control panel makes the simulation safe as well with by preventing the engine from being started in simulation operation mode.

\subsection{OPERATING MODES OF THE VEHICLE CONTROL PANEL}

The vehicle control panel installed on the Audi TT Coupe has the following operating modes.

\section{Normal mode}

In normal mode the vehicle operates 'normally', i.e. like a conventional road vehicle. This mode is safe: the additional electronic devices installed subsequently are isolated galvanically from the original electronic system of the vehicle. This mode is used for regular travelling. If the emergency switch placed in the middle of the vehicle console is pushed, the system returns into this mode. 


\section{Sensor test mode}

In the sensor test mode a simple and quick test of the control panel and the communication system can be run without any other devices (e.g. a PC). In this mode, the accelerator and brake pedal position, the steering wheel angle and the selected gear are displayed on the vehicle dashboard.

\section{Demonstration mode}

In demonstration mode, the embedded software of the control panel runs a demo which demonstrates the main outputs (e.g. dashboard functions and sound generator) of the gateway without any additional devices.

\section{Autonomous simulation mode}

In this mode, the control panel simulates a simplified vehicle model in order to test the main functions and the communication of the simulation system without any additional devices such as a simulation PC. The accelerator and brake pedals, the steering wheel, the transmission selector lever and the simulation function of the dashboard can be checked easily in this operating mode; the sound generator provides the engine sound, as well.

\section{PC simulation mode}

The PC simulation mode is the most relevant operating mode in the vehicle simulator system. In this mode the vehicle is used as an HMI of the simulation run on the simulation PC. Furthermore it provides a feedback of some information to the driver by displaying the vehicle speed, engine rpm, etc. on the dashboard. The simulation mode is safe: the vehicle engine cannot be started in this mode. The vehicle control panel transmits the following signals to the simulation PC:

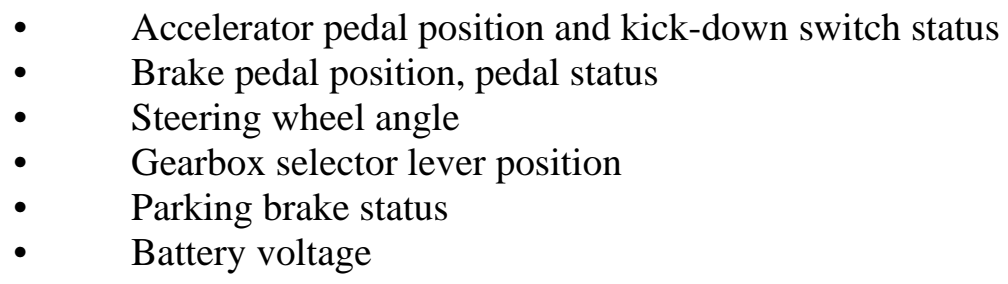

The signals received from the electronic system of the vehicle are read and transmitted continuously by the control panel; the most important signals (e.g. pedal and steering wheel positions) are sent with $10 \mathrm{~ms}$ sampling time and the less important signals (e.g. engine temperature and battery voltage) with 50-100 msec. The duplex CAN gateway can also receive CAN messages from the simulation PC and the relevant information is displayed on the vehicle dashboard. The MATLAB/Simulink simulation calculates the motion of the vehicle and the following signals are sent back to the vehicle control panel in order to be displayed on the dashboard in quasi real-time:
- $\quad$ Vehicle speed
- $\quad$ Engine speed
- $\quad$ Selected gear
- $\quad$ Engine coolant temperature
- $\quad$ Other indicator or error lights

In PC Simulation mode the engine and tire sound are generated by the CarSim, and the sound signals are fed into the original audio system of the vehicle. The system provides realistic driving experience while the driver is sitting in a real car; see Figure 5. 


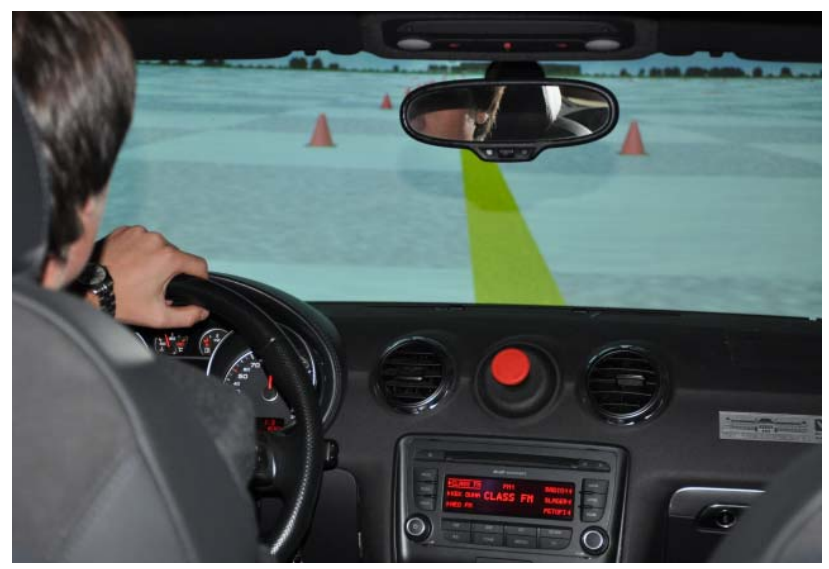

Figure 5: Full in-car driving experience

\section{Measurement mode}

In the measurement mode the vehicle operates in the same way as in normal mode but the vehicle control panel still has the contact with the electronic system of the vehicle. In this mode, the vehicle control panel functions as a gateway; it reads the CAN messages on the five separate CAN buses of the vehicle and the additional sensor modules, then transmits the relevant messages to the PC CAN interface card. For safe operation, it is forbidden to send any messages towards the vehicle. The structure of the measurement system is shown in Figure 6 . The PC logs the CAN data received by the CAN interface card.

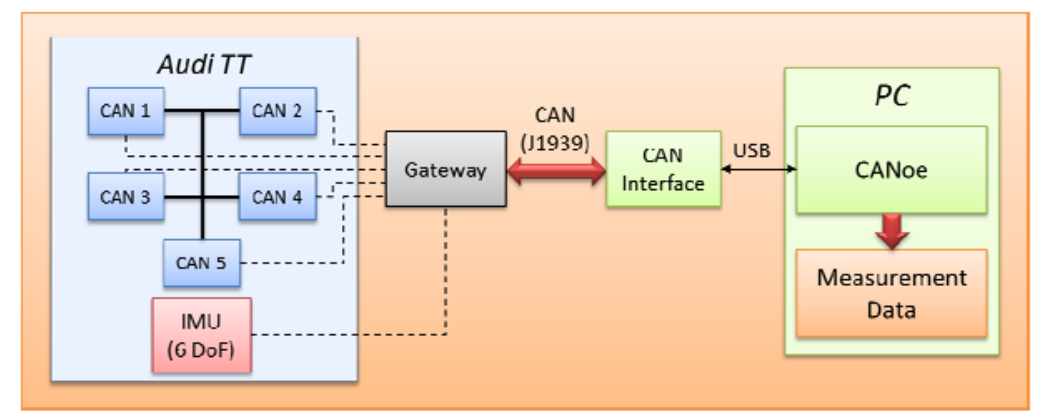

Figure 6: The structure of the measurement system

\section{SIMULATION SOFTWARE}

The vehicle model, the road, the environment and the scenario of the simulation are built in CarSim. CarSim is a stand-alone simulation software which contains a number of validated complex vehicle models of passenger cars, racecars, light trucks and utility vehicles. Custom vehicle component models can be built as well or even replaced by external custom models; e.g. models built in Simulink or even written in C programming language. The software is able to use its own solver and cooperate with external softwares such as MATLAB. Other software components are available for the basic CarSim environment; modules for modeling flexible chassis, for using dSpace AutoBox for hardware-in-the-loop (HIL) simulations and so on. For the vehicle simulator the basic CarSim is extended with a Driving Simulator. This extension module ensures the real-time running and visualization of the scene. The vehicle model generated by CarSim is embedded in a Simulink model. 
The Simulink model also contains the communication functions which receive and transmit the CAN messages by the CAN interface card. The positions of the pedals, the steering wheel and the transmission selector lever are fed into the vehicle model. Any signals of the vehicle model (e.g. positions, velocities and accelerations) can be read and used by the control systems. Vehicle control systems, designed in MATLAB/Simulink, are added to the simulation model. Figure 7 shows the screenshots of the simulation softwares.

The vehicle simulator uses mainly a custom vehicle model which is built for simulating the real Audi TT. Other built-in CarSim vehicle models are used for testing vehicle control algorithms as well. The purpose is to build a more sophisticated vehicle model for the simulator which converges to the real car. The validation of the vehicle model is carried out by measurements.

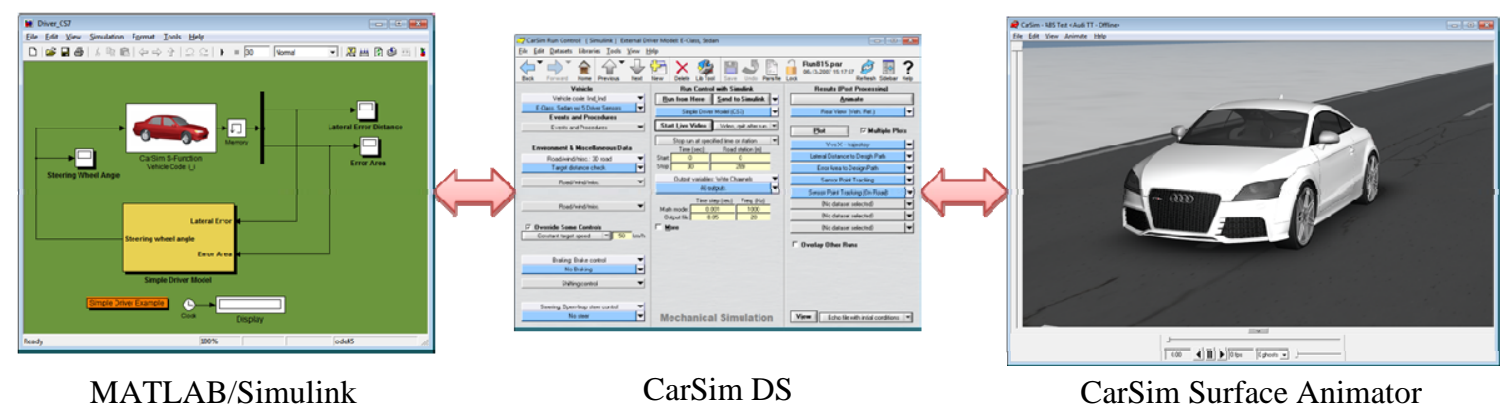

Figure 7: Screenshots of the simulation softwares

\section{EDUCATION PURPOSES OF THE SYSTEM}

When the topic of 'Systems and Control Theory' is taught the theoretical methods can be illustrated in a simulation environment, see $[1,2,3]$. An important topic is the demonstration of the control design methods applied in the industry [4]. For example, the operation of an ABS/ESP or a ROP (Roll Over Prevention) control can be presented. The effect of changes in the control parameters can be further analyzed. Moreover, the simulation system supports the training of the driver. Different driver behaviors can be simulated and analyzed. Different responses to emergencies during vehicle maneuvers can be compared and the cause-effect relationships can be explored. In this way the appropriate response can be practised.

Using the simulator system during lectures of "Automotive Communication Systems" students can understand how the CAN networks of vehicles are structured and what residual bus simulation means in practice. As the complete CAN communication to the instrument panel is taken over by the control panel in simulation mode, high level understanding of the operation is demonstrated.

\section{RESEARCH ACTIVITIES OF THE SYSTEM}

The vehicle simulator is also a basis for research activities. Various journey scenarios can be generated by the simulation system. The advantage of the system is that besides measuring various signals, i.e., the steering angle, the positions of the accelerator and the brake pedal or the gear level, in principle any signals can be monitored during the simulations. In this way signals which are not measurable in practice can be obtained for analysis purposes. Below some research directions are presented. 


\section{A. Trajectory tracking}

The purpose of trajectory tracking is to follow road geometry at a predefined velocity and guarantee the road stability of the vehicle simultaneously. Several vehicle control components can be applied such as such as the brake or the steering. In the control design the interaction and priorities between the controllers must be taken into consideration. The time responses of the trajectory tracking algorithm based on 4WS (Four Wheel Steering) implemented on the simulator are illustrated in Figure 8.
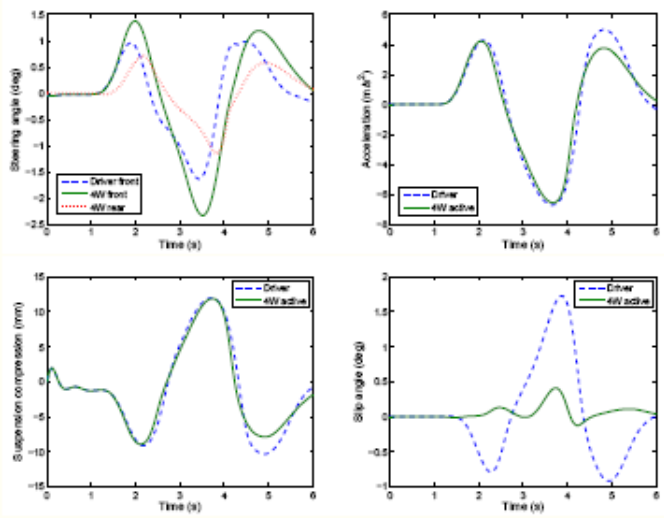

Figure 8: Example of a trajectory tracking control algorithm tested on the vehicle simulator (steering angle, acceleration, suspension compression, slip angle)

\section{B. Mu-split control}

When hard braking is taking place on a road on which friction significantly differs between the left and the right wheel paths (mu-split), the vehicle may rotate over the wheel path. There are several control methods which are able to handle this emergency. As an example the time responses of the operation of the brake control are depicted in Figure 9.
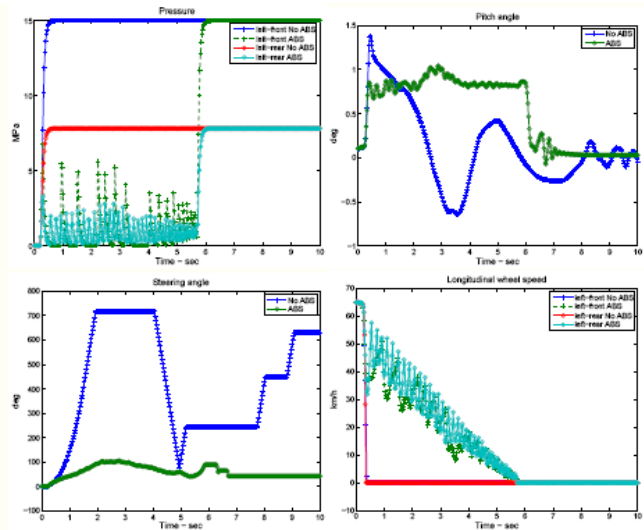

Figure 9: Example of a vehicle stability control algorithm tested on the vehicle simulator (brake pressures, pitch angle, steering angle, longitudinal speed)

\section{Driver assistance systems}

Driver assistance systems usually support the driver activities, since they have more information about the vehicles, the road (adhesion) and the environment (traffic signs, speed limit). A general solution is that the driver requirements are built into the automatic control 
system, e.g. sport and comfort style. In a typical sport mode the steering responses are sharper, the ride is firmer and the driving response improves. In comfort mode, these features are considerably less sharp. An example of the vehicle control algorithm can be seen in Figure 10 .
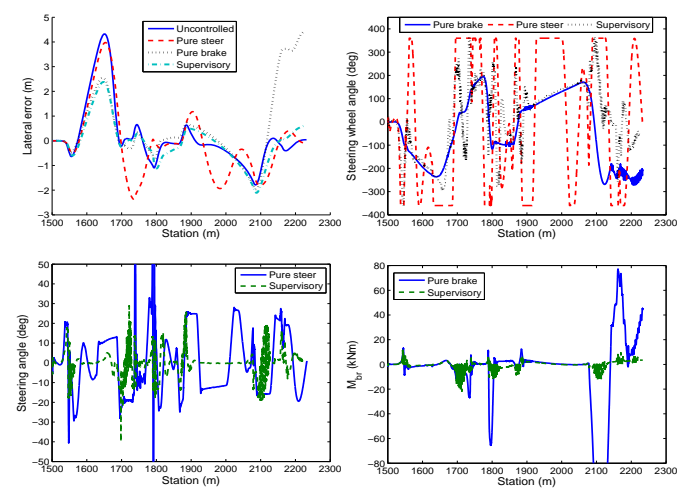

Figure 10: Example of an integrated vehicle control algorithm tested on the vehicle simulator (lateral error, steering wheel angle, steering angle, brake yaw moment)

\section{CONCLUSIONS}

A real-vehicle-based simulator has been developed and tested, with the advantage of simultaneous use in education and scientific research on virtual or real test tracks. The unique feature of this simulator system is that the HMI of the simulator is a cockpit of a real car, which can also be driven as a normal vehicle on the real test track. The significance of such a simulator in university education is that the students are much more motivated in this environment, while the researchers are enabled to implement autonomous driving functions on the running vehicle.

\section{ACKNOWLEDGEMENT}

The development was supported by the project of Vehicle technology, transport and logistics (JKL) Research University Program by grants TÁMOP-4.2.1/B-09/KMR-2010-0002 and TÁMOP-4.2.2.B-10/1-2010-0009.

\section{REFERENCES}

[1] Gillespie T., “Fundamentals of vehicle dynamics”, Society of Automotive Engineers Inc., 1992.

[2] H. B. Pacejka. Tyre and vehicle dynamics. Elsevier Butterworth-Heinemann, Oxford, 2004.

[3] R. Rajamani, "Vehicle dynamics and control,” Springer, 2005.

[4] A. Trachtler. Integrated vehicle dynamics control using active brake, steering and suspension systems. International Journal of Vehicle Design, 36:1-12, 2004. 